\title{
Drying methods of the printing inks
}

\begin{abstract}
Accelerating the transition to post-print processes needed in the printing industry and shortening the time the product's release time is closely related to the drying time of the ink film. The drying of fluid ink on the surface of the print substrate, transforming from liquid to solid occurs physically and chemically in several ways. Drying systems can be functional alone on the surface of the printing substrate for an ink film or depending on the chemical content of the ink and the properties of the printing substrate, drying can be achieved at the same time with more than one system. Recently, in order to reduce climate, environmental and health impacts and with the development of technology, significant changes are also being experienced in the printing industry and preferences are changing. In this study, more environmentally friendly LED UV and microwave drying systems that save time and energy together with existing basic drying systems such as absorption, evaporation, oxidation-polymerization and conventional UV used in the printing industry are examined. The advantages of different drying systems to the printing industry, preferred drying systems and new studies on this issue have been evaluated.
\end{abstract}

\section{KEY WORDS}

Drying system, UV-curing, LED-UV, microwave drying, radiation curing

\author{
Ashraf Abd El-Rahman \\ Elsayed Saad ${ }^{1}$ \\ Cem Aydemir ${ }^{2}$ \\ Samed Ayhan Özsoy ${ }^{3}$ \\ Semiha Yenidoğan ${ }^{2}$ \\ ${ }^{1}$ Independent Consultant in \\ Printing and Media Technology \\ European and Arab Region, \\ Wuppertal, Germany \\ ${ }^{2}$ Marmara University, \\ School of Applied Science, \\ Department of Printing \\ Technologies, Istanbul, Turkey \\ ${ }^{3}$ Istanbul University- Cerrahpasa, \\ Vocational School of Technical \\ Sciences, Printing and Publication \\ Technologies Program, \\ Istanbul, Turkey
}

\author{
Corresponding author: \\ Cem Aydemir \\ e-mail: \\ cemaydemir@marmara.edu.tr
}

\section{Introduction}

The visual quality of print results is substantially based on the optimal level of ink merging with a substrate, particularly the drying and stabilization process of ink on the substrate (Aydemir et al., 2019). Drying is when the ink changes from a liquid state to a solid state after it is transferred to the printing substrate through the rollers and printing plate. The complete drying process required for the ink film is very important for finishing processes such as lacquering, coating, folding and cutting post-printing. (Aydemir, 2010).

In printing, the most important element is to obtain the correct substrate and ink combination (Aydemir \& Yenidoğan, 2018). A physicochemical interaction 
between paper and printing ink greatly determines the spreading of wet ink, as well as setting and drying (Aydemir, 2016). In the process of fluid ink settling and absorption onto the paper surface, the surface characteristics of the paper are extremely important. Depth and width differences on the paper surface can affect the quality parameters of the ink film, such as ink settling on the paper, print density, print gloss, and color (CIE L*a*b*) (Aydemir et al., 2021). Ink's structure and solvents are also determinants in the drying process of the ink. In addition, air, temperature, and humidity have a major influence on the drying speed of the ink.

With the increasing use of complex surfaces and inks, the need for different drying techniques has increased in the printing process. Sensitivity to environment and human health has also caused changes in drying systems (increasing use of water-based inks instead of solvent-based inks, etc.). Drying in the printing process generally takes place by the penetration or evaporation of the ink solvent into the printing substrate and the chemical change of the ink. A combination of two or more drying systems can be used to ensure final drying of the ink film. Some inks dry by a combination of two or more drying mechanisms, such as "penetration into the printing substrate" and "air oxidation" (McKinney, 1995; Brancher, 2019). For example, in the web-offset heat-set drying process, some of the solvent oils evaporate from the ink, while some are absorbed by the printing substrate. The remaining part of the ink undergoes oxidation- polymerization and drying occurs.

\section{Drying Methods of Printing Inks}

Drying of the printed substrates is an important issue for the printing industry. The ink is expected to show good performance in terms of fluidity and adhesiveness during transfer from the ink fountain to the printing substrate during printing, and to dry after transfer to the printing substrate. Fast or slow drying creates difficulty in application. If the ink cannot penetrate fast enough into the paper, there can appear problems during the printing process of trapping issues, or, after the printing process, blocking, smearing, set-off to the back of the next sheet. If on the other hand the ink sets too fast, penetrates too fast into the paper, other problems can show up, like strike-through, trapping again and picking.

Drying of printing inks depends on the following factors:

- Printing method (offset, gravure, flexography etc.) - Inks type (liquid, paste, water-based or organic solvent-based, radiation curing inks, etc.)

- Ink rheology (viscosity, flow, tack)

- Speed of printing machines

- Printing substrates (paper, foil, plastic etc.) and their properties
- The characteristics of the dryer systems (hotair, cured systems, IR, etc.) (Saad, 2007).

The drying of the ink film is accomplished by physical (penetration, evaporation) processes and chemical reactions (oxidation or polymerization) or a combination of both, depending on the ink formulation and the properties of the printing substrate.

The main drying methods are as follows;

- Absorption drying

- Evaporation drying

- Oxidation and polymerization drying

- Radiation (Ultraviolet-Infrared-Microwave)

In general, gravure, flexography and web-offset inks are by evaporation, sheet-fed offset inks are by oxidation-polymerization and cold-set inks are dried by absorption (Saad, 2007).

\section{Absorption Drying}

Printing inks consist mainly colorants (dyes or pigments), binders (natural resins, artificial resins, or plastics), solvent or solvent blend and additives. Drying by absorption occurs when the liquid components (predominantly solvent part) in the printed ink film are absorbed by the pores of the printing substrate. Absorption is an interaction between ink and printing substrate (Figure 1, 2). Generally, this type of drying method is depending on the carrier viscosity of the printing ink, the vehicle (binder) and the absorption capacity of the substrate (Saad, 2007). With absorption, drying occurs mainly on absorbent surfaces such as paper and cardboard, and several properties of these substrates manage the absorption of ink (Huber, 2013).

Ink film

Paper

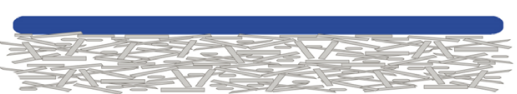

» Figure 1: Newly printed ink film on the top surface of the paper

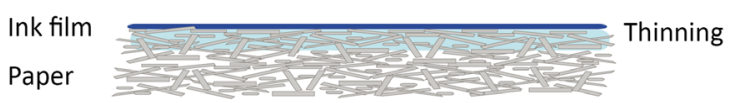

» Figure 2: Absorbing ink film on the top surface of paper

The ink-absorption capacity is an important factor for evaluating the printing of paper (Dong, et al., 2020). The liquid absorption ability of paper and cardboard is based on micro capillarity (Imamoglu et al., 2013). In this drying technique, the printing substrate surface absorbs the solvent (solvent or mineral oil, etc.) in the wet ink film and separates it from the resin and pigment (Aydemir, Yenidoğan \& Özsoy, 2020). Paper or cardboard acts as a filter with this feature (Aydemir \& 
Özakhun, 2014). Since the solvent is not fully compatible with the resin and pigment mixture in terms of its chemical structure, it dissociates and penetrates into the fine capillary fiber tubes of the paper horizontally and vertically at a very low speed (Figure 3) (Brancher, 2019). Thus, the released resin and pigment allow the formation of a hard and solid layer on the surface. A fully solidified ink film can sometimes be formed in 6 to 7 days. The absorption ability of the paper surface plays an important role in the drying of the ink (Brancher, 2019).

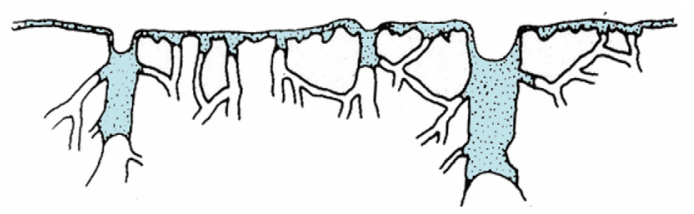

» Figure 3: Capillary structure of paper and absorption of ink

The pore structure of the paper is decisive for the absorption process of ink. If the pore diameter of the paper is small enough, only oil enters the paper, while the pigment and the rest of the vehicle remain on the surface. If the pore diameter of the paper is too large, the pigment and vehicle also penetrate the paper, which leads to a decrease in the color strength of the printed ink. Drying by means of absorption occurs faster and more intensively on its uncoated surfaces, while the absorption of ink on coated paper surfaces is quite slow and low in quantity.

Especially in cold-set offset printing, wet ink film dries by being absorbed by paper or penetrating into paper fiber body (Tsigonias et al., 2010). Absorption into the paper body also occurs in water-based and solvent-based inks. The viscosity of the ink is as effective as the pore structure of the paper in the absorption of the ink (Sunnerberg \& Larsson, 1987).

\section{Evaporation Drying}

The fast evaporating solvents in the ink composition separate from the ink after printing and evaporate. The remaining binder combines with the pigment and creates a hard color layer on the printing surface. The evaporation of the liquid phase causes the remaining ink components to come into more contact with each other and create a continuous film (Figure 4) (Brancher, 2019).

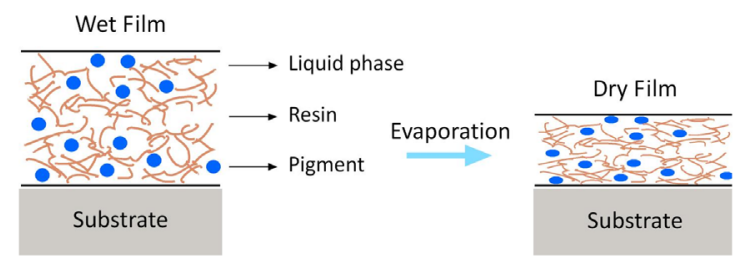

» Figure 4: The appearance of pigment particles after evaporation of the ink
The reach of liquid ink to the desired density depends on the evaporation time of the solvent inside. As the liquid phase evaporates, the viscosity of the ink increases and the ink film begins to change from liquid to solid (Sappi Printer Technical Service, 2012). It then gives a dry ink film that adheres well to the print substrate (Brancher, 2019).

Solvents used in gravure, flexography and screen printing inks evaporate at room temperature. High temperature and heat energy are needed to remove the oil-based solvent used in heat-set offset printing inks (Aydemir, 1999). In the heat-set printing drying process, the paper is exposed to oven drier temperature of about 120 $150^{\circ} \mathrm{C}$ so that the solvent (mineral oils in the boiling range of $85-120^{\circ} \mathrm{C}$ containing aliphatic hydrocarbons) in the printed ink film can evaporate (Aydemir, Akgül \& Tutak, 2020; Smyth, 2003). The long hot-air dryer is used to blow hot-air over both sides of the fresh print via nozzles (Tsigonias et al., 2010). The local temperature rise in the dryer causes the ink solvent to evaporate.

\section{Despite the above-mentioned facts, the evapo- ration drying method still has some problems:}

- A longer time is required to dry waterbased inks with evaporation methods. Because the drying of water-based inks is 4.5 times slower than solvent-based inks.

- Alcohol is used to improve the wetting and drying properties of water-based inks. Alcohol use (if there is an ammonia and amine group) causes environmental pollution.

- Most of the ink solvents that evaporate during the printing process are released into the atmosphere. Evaporating solvents are known to severely damage the ozone layer (Aydemir \& Özsoy, 2020).

- During the evaporation of organic solvent-based inks, high capital investments are required in order to prevent the pollution caused by the waste air from the dryer exhaust in the atmosphere.

- Energy consumption is high.

- Drying systems for water-based and oilbased inks require considerable space to achieve complete drying.

- In flexography printing, excessive heat may cause the visco-elastic properties of plastic substrates to change.

- In heat-set web-offset printing, the possibility of overheating of the paper line can cause changes in the fiber size of the paper, with moisture loss in the non-printed areas of the paper substrate. Therefore, printing finishing processes are getting more and more difficult (Saad, 2007).

- With high heat, the printing substrate color and printing colors may change. 


\section{Oxidation and Polymerization Drying}

This type of drying system depends on the chemical reaction between the oxygen in the atmosphere and the drying oil in the components of the carrier system (Saad, 2007). Except for the part (liquid phase) that is absorbed or evaporated after the ink is printed, the content that cannot enter the capillaries and remains on the paper surface (unsaturated vegetable oils such as linseed oil, soybean oil, tung oil, resins and pigments) polymerizes when exposed to atmospheric oxygen. (Figure 5) (Huber, 2013; PrintWiki, 2018). The oxygen in the air adds to double bonds of the drying oil molecules to form hydroperoxides (Saad, 2007). Thus, a rigid ink film with a thickness of 2- 3 micrometer is formed on the surface of the print substrate (Saad, 2007). In other words, the combination of the binding group molecules within the ink with the oxygen of the air enables the formation of new and larger molecules (molecular change). Also, since most molecules have more than one reactive site (i.e. double bonds), some cross-links form by forming a network (Saad, 2007) and the ink changes from a thin and soft state to a solid state. The drying of (sheet-fed) offset inks on the surface of less absorbent and non-absorbent printing papers usually occurs in this way.

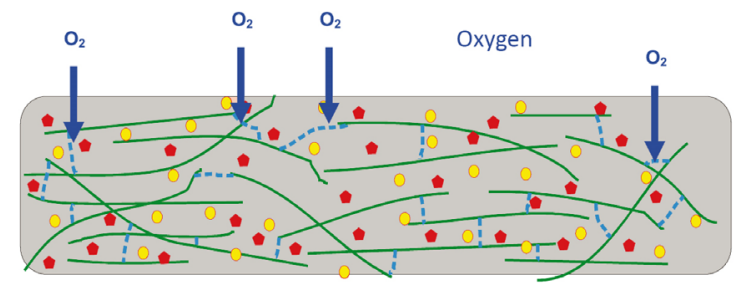

» Figure 5: Oxidative drying in offset printing

Following the physical drying process, the chemical drying time varies according to the oxygen uptake of the working environment and is accelerated by a heating (Smyth, 2003). Through initiators or catalysts (metal dryers such as cobalt, lead, manganese salt), oxygen in the air reacts more easily with the binding part of the ink and polymerization accelerates.

\section{Radiation Drying}

In cases where physical and chemical drying is insufficient, radiation and ink drying systems can be used. There are several types of radiation used to dry inks. These include ultraviolet radiation (UV), infrared (IR), microwave (MW) and radio frequency (RF). Each drying method determines the ink chemistry and the nature of the print ink used (Leach et al., 1988). Radiation drying types are preferred by printing enterprises because they increase the type of substrate and printing speed, accelerate the transition to post-printing processes, prevent drying problems of the ink, and reduce environmental effects (Figure 6).

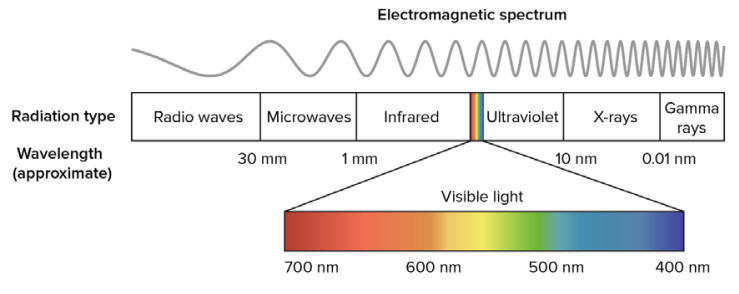

» Figure 6: Radiation drying - Electromagnetic spectrum (Khan Akademy, 2017)

\section{Ultraviolet (UV) Curing}

UV curing is a photo-polymerization process that uses UV energy source (the UV light) to transform a liquid into a solid. With the absorption of the UV energy, the photo-initiators contained in varnish, ink, adhesive or resin in the liquid state produce substances called free radicals, that react with the chemical compounds of the liquid substance, turning it into solid. This process is also called "polymerization" (PhotoElectronics, 2019; Kunwong, Sumanochitraporn \& Kaewpirom, 2011). UV curing is widely used in sheet-fed offset printing, inkjet printing, screen printing and flexography printing where oxidation drying is insufficient, especially in packaging applications (Smyth, 2003; Leach et al., 1988).

For UV curing, UV-curable inks whose binder and solvent molecules react to UV radiation and form a fixed film layer in a short time must be used. These inks contain photo-initiators that absorb UV radiation and thus generate highly active chemical compounds known as free radicals (Argent, 2008).

UV inks is cured by a chemical polymerization reaction initiated by exposure to UV radiation and can therefore only be used in printing machines with UV curing systems (Aydemir, 1999; Brilliant Universal Limited, 2012). UV systems consist of beam source (lamp) and reflectors as in IR systems. UV dryers can be added to the output of printing machines or can be placed between printing units for intermediate drying (Argent, 2008).

UV curing uses light energy to initiate polymerization (Argent, 2008). Liquid ink is exposed to UV radiation by passing under the metal halide or mercury vapor arc lamps in the machine immediately after printing (Brancher, 2019). Strong UV radiation hits the photo-initiators in the ink and activates it, and the photo-initiators become macromolecules under the effect of the radiation. Liquid ink begins to harden. At the end of the reaction, cured or polymerized, a solid layer is formed that combines the pigment (Figure 7) (Smyth, 2003).

The curing speed of the ink; the spectral distribution of the lamp depends on the reactivity of the printing ink, the radiation and radiation distribution in the curing 


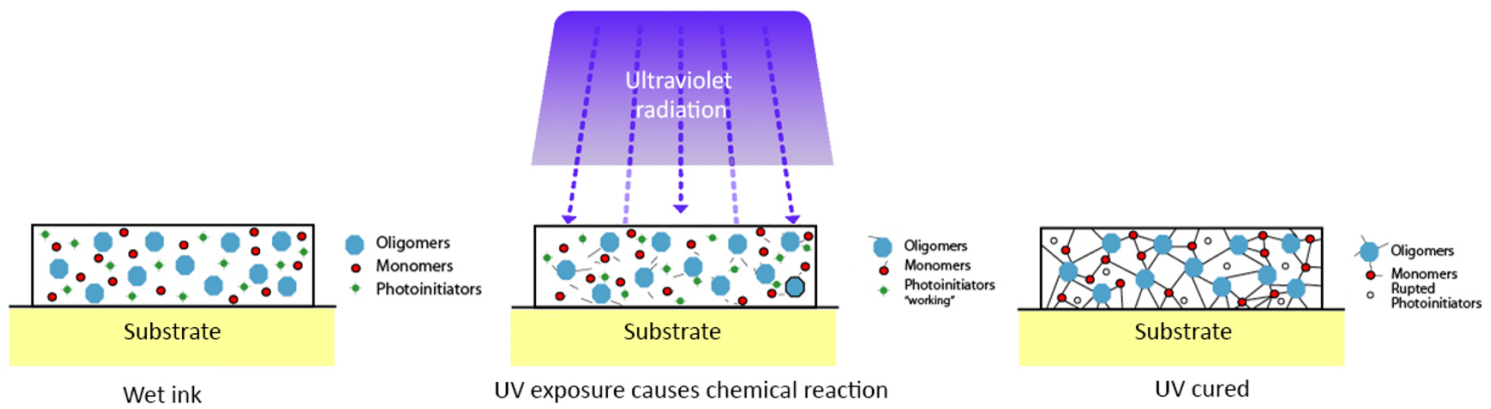

» Figure 7: Ultraviolet curing

plane, the thickness of the ink, the oxygen concentration (air or inert), the coating temperature conditions (Mehnert, 1999).

Since different photo-initiators require different UV wavelengths, radiation from the source and photo-initiators must be matched to ensure polymerization (Hung, Wimberger \& Mujumdar, 2006). For this purpose, UV lamps radiate light at different UV ranges to have maximum drying efficiency.

The UV radiation spectrum is located in a series of wavelengths shorter than visible light and can be divided into 3 parts:

- UV-C (200-280 nm) activates photo-initiators and lets the surface dry out.

- UV-B (280-315 nm) continues polymerization reaction.

- UV-A (315-380 nm) provides deep curing (Figure 8) (Brancher, 2019).

\begin{tabular}{|c|c|c|c|}
\hline Rayons X & UV & Light Visible & IR \\
\hline UV-C & UV-B & UV-A \\
\hline $200 \mathrm{~nm}$ & $280 \mathrm{~nm}$ & $315 \mathrm{~nm} \quad 380 \mathrm{~nm}$
\end{tabular}

» Figure 8: Ultraviolet wavelengths

The power output and condition of lamps and reflectors significantly affect the amount of UV light energy the ink is exposed to (Argent, 2008).

Regardless of the type of dryer or ink used, it is very important to check the power rating, nature and condition of the lamps during printing, the printing speed and the compatibility of the amount of ink consumed.

If the inks do not dry adequately, several operations can be done, such as checking the efficiency and working hours of each lamp, and slowing down the printing speed (Brancher, 2019).
Advantages of the UV system:

1. The lack of solvent in UV ink makes UV curing an attractive alternative in situations where solvent emissions need to be reduced (Brilliant Universal Limited, 2012).

2. Print stability and print quality are very good in UV curing because the chemical reaction does not start during drying until energy is applied. Therefore, there is no volatile organic compounds (VOC) problem (Argent, 2008).

3. The UV curing process is very fast, usually completed within fractions of a second. This means less space required. Shelf or secondary drying processes are eliminated with UV curing (Brilliant Universal Limited, 2012).

4. Since the lamp reflectors in the dryer are cooled by air or water cooling equipment, the effect of the heat rays coming to the printing substrate decreases compared to the IR technique. This allows printing of certain heat-sensitive materials (PVC, polyester, etc.) of certain thickness.

\section{LED-UV Curing}

In the traditional UV curing process, quartz discharge lamps have been used for many years, and these lamps contain lead, mercury and cadmium metals (PhotoElectronics, 2019). The disadvantages of conventional UV systems are that they produce ozone, need exhaust systems to maintain air quality, use a lot of energy, and emit a lot of heat. Also, the disposal of used mercury arc vapor lamps is problematic. Because mercury is a toxic metal, it has attracted the attention of environmental regulators.

LED-UV are definitely the innovation of the future, offering many advantages in curing and drying industrial processes. For this reason, its use is increasing day by day. With the development of LED technology, changes are also experienced in UV curing systems. The LED-UV curing system uses diodes that convert electrical current into light. When the electrical current flows through an LED, it gives off IR or UV radiation. The UV light causes chemical reactions in the molecules within the liquid, forming chains of polymers until the liquid becomes a solid. 
This process is a new technology that was designed to provide solutions to many of the issues found in conventional UV curing and heat-set drying (DoctorUV, 2018).

The LED-UV curing system, which is preferred in sheetfed offset lithography and digital printing machines, allows printing on substrates with lower thickness and different heat-sensitive because it emits less heat than conventional UV systems. It also reduces electricity consumption and can contribute to a reduction in VOC (Mirković, Medek \& Bolanča, 2019). LED-UV systems are more environmentally friendly due to odorless production, less heating and lower energy consumption. With these advantages, it provides an important alternative to conventional UV curing systems for solidifying inks, lacquers and adhesives (DoctorUV, 2018).

\section{Infrared (IR) Drying}

Infrared (IR) drying is a kind of radiant heating. In this drying system, no mechanical effects such as highspeed hot-air jets are required for heating. IR radiation may transfer large amounts of energy in short time. The energy emitted by IR radiation is directly used for heating the wet ink film (Saad, 2007). In this system, heat energy is generated when the ink film absorbs sufficient IR light energy. At high temperatures oxidation, penetration, evaporation and polymerization mechanisms all accelerate (Leach et al., 1988).

The wavelength spectrum of the radiation depends on the nature and temperature of the heat source. The wavelength range of thermal radiation is $0.75-1000 \mu \mathrm{m}$ within the spectrum. IR radiation is conventionally classified as shortwave $(0.75-3.00 \mu \mathrm{m})$, medium-wave $(3.00-25 \mu \mathrm{m})$, and long-wave $(25 \mu \mathrm{m}-1000 \mu \mathrm{m})$ (Figure 9), (Selim et al., 1997).

As the wavelength of the IR radiation is too long (much longer than the wavelength of UV radiation), the energy of their photons is too low (it is much lower than the energy of UV photons) to achieve any photochemical reactions. This means that, with IR radiation only a heating of the printed substrate is reached (Saad, 2007).
The most common current applications of IR drying in printing are dehydration of ink films (Ratti \& Mujumdar, 2006). IR technology is mostly used to solve the drying problem of water-based inks, varnishes, lacquers and adhesives that cannot be evaporated. The spectrum of the medium wave radiator is very well matched to the absorption line of water, so it is highly efficient and economical in water-based ink drying (Jingxiang et al., 2019). Shortwave IR radiators, on the other hand, are effective in drying thick wet ink films due to their high penetrating power. Maximum drying sensitivity of offset printing inks is in the medium wavelength range of 3-3.6 $\mu \mathrm{m}$. It is important to adjust the IR wavelength for the absorption of the inks to substrates (Saad, 2007).

IR dryers are used in newspaper printing only to stimulate ink adsorption, thereby facilitating printing with higher ink density, that is, with higher ink application (Tsigonias et al., 2010). UV and IR drying systems can be used for drying inks as well as protective and decorative over-print varnish and lacquer applications.

\section{Microwave (MW) Drying}

Microwave (MW) drying is a technique based on electromagnetic waves that generate heat directly inside the ink film. MW radiation is a part of the electromagnetic spectrum ranging from $300 \mathrm{MHz}$ to $300 \mathrm{GHz}$ (Waghmare et al., 2021). This system effectively, quickly and efficiently dries the water-based inks on the paper.

Various liquid materials in printing such as inks, varnishes, lacquers, and adhesives contain high ratios of water. (Aydemir, Altay \& Akyol, 2021). Water and other polar solvents are easily heated by microwave energy. Polar solvent molecules act like small magnet. The movement of molecules then generates heat. Under the action of the microwave electromagnetic field, the heated medium molecules become polarized molecules with positive and negative charges. Since the microwave field changes rapidly, solvent molecules oscillate and try to align with the field. MW are non-ionizing, they can interact with dielectric materials to generate heat by agitating molecules in an alternating electromag-

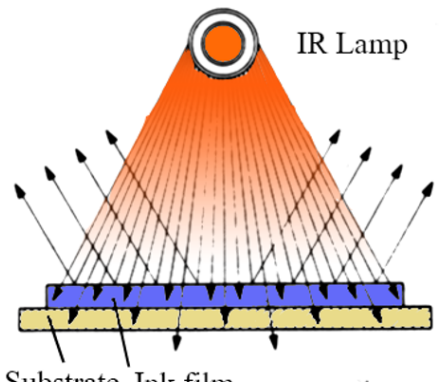

Substrate Ink film

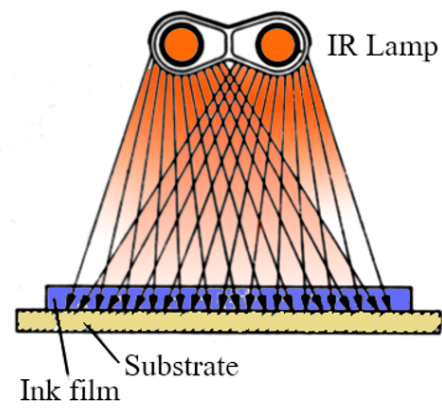

Medium-wave

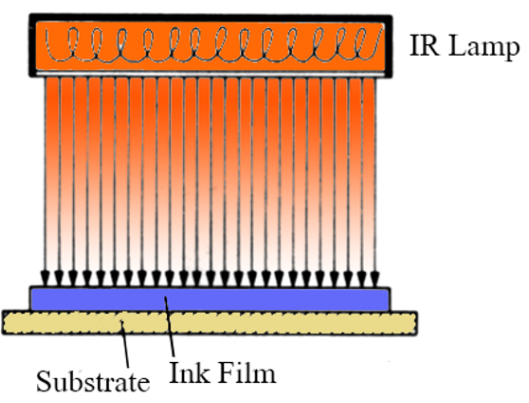

Long-wave

» Figure 9: Short, medium, long wavelength drying 
netic field. While solvent-based inks are widely used in flexography, gravure and screen printing systems, the use of water-based inks is limited (Aydemir \& Özsoy, 2020). Water-based inks create a drying problem due to the low evaporation rate of water (McKinney, 1995). Despite such problematic aspects of water-based inks, use to the instead of solvent-based inks is still greatly encouraged due to environmental and health concerns.

Infrared radiation or other hot-air drying methods, require a long drying period and large amounts of energy. Besides that, conventional drying mechanisms lead to, in certain cases, insufficient drying which results in misprints and added cost (Tsigonias et al., 2010). Using a microwave-assisted drying system has environmental benefits compared to a conventional drying system (Jingxiang et al., 2019). In addition, MW drying is known to provide extremely high energy savings compared to existing conventional drying technologies, although equipment costs are high (Sharma, 2015).

Marios et al. compared several drying methods common to water-based inks, hot-air drying, IR drying, MW drying speed and energy consumption (Tsigonias et al., 2010). The results proved that MW drying has distinct advantages in terms of both drying speed and energy consumption. In the study conducted by Marios, the energy consumption of MW drying was much less than that of hot-air drying.

\section{Radio Frequency (RF) Drying}

The principle of the RF drying method is very similar to MW drying. In this system, an alternating electric field is created between two electrodes. The material to be dried (for example glue, inks and paper, carton or others) is conveyed between the electrodes where the alternating electric field causes polar molecules in the material to continuously re-reorient themselves to face opposite poles such like the way bar magnets behave in an alternating magnetic field. The friction resulting from molecular movement causes the material to rapidly heat throughout its entire printed ink film (Trembley \& Loring, 1969).

\section{Conclusion}

It is important for ink manufacturers to test the placement behaviors of the ink and for paper manufacturers to control the absorption behaviors of the paper to improve the drying process. In this study, the drying methods used in the printing processes were evaluated in terms of printing substrate, ink and printing systems. According to this;

Drying systems can be functional alone on the surface of the printing material for an ink film, or multiple systems can be active simultaneously, depending on the chemical content of the ink and the properties of the printing substrate. High-speed hot-air drying, IR drying, UV curing, MW drying and other conventional drying methods can be used together to increase the drying speed.

UV-curable inks and coatings contain low amounts of or no VOC. Therefore, UV curing systems can be preferred instead of evaporative drying systems using solvent-based inks. However, UV curing must be designed and used to meet all safety requirements of personnel and the environment due to biological effects on the skin and eyes and hazards from ozone, nitrogen oxide (NOx) and other by-products.

UV ink and UV over-print lacquers can cause large stains that cannot be easily removed with conventional de-inking methods and can be seen on recycled paper. This situation should not be overlooked in the selection of ink and drying system.

The MW drying method can be preferred in prints made with water-based ink and in cases where the printing substrate properties are not desired to be adversely affected. Because studies have shown that MW drying does not damage the ink-free areas of the paper. With a microwave-assisted drying system, both environmental benefits and energy and cost efficiency can be achieved compared to a conventional drying system.

Finally; Excessive ink consumption in the printing process will have negative effects on the economy and the environment due to higher consumption of resources to be used in drying energy. For this reason, the environmental sustainability of printing can be achieved by keeping ink consumption at an optimum level..

\section{References}

Argent, D. (ed.) (2008) Drying Part 3: Paste Inks. Available from: https://www.pffc-online. com/print/inks/6097-drying-part-paste-0401 [Accessed 20th February 2021].

Aydemir, C. (1999) Determination of the ideal drying temperature of heat-set web offset inks by vapor pressure method. PhD thesis. Marmara University Institute of Natural and Applied Sciences.

Aydemir, C. (2010) Time-dependent behavior of a sessile water droplet on various papers. International Journal of Polymeric Materials. 59 (6), 387-397. Available from: doi: 10.1080/00914030903538470

Aydemir, C. \& Özakhun, C. (2014) Printing Materials Science. Istanbul, Marmara University Publisher. pp. 262-264.

Aydemir, C. (2016) A study on the printability properties of alkali-sized recycled papers. Science and 
Engineering of Composite Materials. 23 (5), 565571. Available from: doi: $10.1515 / \mathrm{secm}-2013-0266$

Aydemir, C. \& Yenidoğan, S. (2018) Light fastness of printing inks: A review. Journal of Graphic Engineering and Design, 9 (1), 37-43. Available from: doi: 10.24867/JGED-2018-1-037

Aydemir, C., Karademir, A., Imamoğlu, S., Altay, B. N., Fleming, P. D. \& Tutak, D. (2019) Investigation of the evolution of hydrophobicity and wettability of paper in multi-color printing process. Cellulose Chemistry and Technology. 53 (7-8), 787-794. Available from: doi: 10.35812/CelluloseChemTechnol.2019.53.77

Aydemir, C., Akgül, A. \& Tutak, D. (2020) Effects of oven drying and polydimethylsiloxane (Pdms) emulsion coating on heat-set printing quality. Cellulose Chemistry and Technology. 54 (5-6), 495-503. Available from: doi: 10.35812/CelluloseChemTechnol.2020.54.50

Aydemir, C., Yenidoğan, S. \& Özsoy, S. A. (2020) Effects of ink consumption on print quality on coated cellulose-based paper surfaces. Cellulose Chemistry and Technology. 54 (1-2), 89-94. Available from: doi: 10.35812/CelluloseChemTechnol.2020.54.10

Aydemir, C. \& Özsoy, S. A. (2020) Environmental impact of printing inks and printing process. Journal of Graphic Engineering and Design. 11 (2), 11-17. Available from: doi: 10.24867/JGED-2020-2-011

Aydemir, C., Altay, B. N. \& Akyol, M. (2021) Surface analysis of polymer films for wettability and ink adhesion. Color Research \& Application. 46 (2), 488-499. Available from: doi:10.1002/col.22579

Aydemir, C., Kašiković, N., Horvath, C. \& Durdevic, S. (2021) Effect of paper surface properties on ink color change, print gloss and light fastness resistance. Cellulose Chemistry and Technology. 55 (1-2), 133-139. Available from: doi: 10.35812/CelluloseChemTechnol.2021.55.14

Brancher (2019) Drying. Available from: http:// www.brancher.com/Drying.html?lang=en [Accessed 15th September 2020].

Brilliant Universal Limited (2012) UV curing technology. Available from: http://www.bul.com.hk/bul/en/technologies/uv_curing [Accessed 20th October 2020]

DoctorUV (2018) UV LED curing systems. Available from: https://www.doctoruv.com/uv-curing-systems/ uv-led-curing [Accessed 10th November 2020].

Dong, Y., Wang, B., Ji, H., Zhu, W., Long, Z., \& Dong, C. (2020) Effect of papermaking conditions on the ink absorption and overprint accuracy of paper. BioResources. 15 (1), 1397-1406. Available from: doi: 10.15376/biores.15.1.1397-1406

Huber (2013) Inkformation Brochure. Available from: https://www.hubergroup.de/fileadmin/_migrated/content_uploads/INKFORMATION_4_en_02. pdf [Accessed 23rd October 2020].

Hung, J. Y., Wimberger, R. J. \& Mujumdar, A. S. (2006) Drying of coated webs. In: Mujumdar, A. S. (ed.) Handbook of Industrial Drying. 3rd Edition. Boca Raton, Florida, CRC Press, pp. 931-951.
Imamoglu, S., Karademir, A., Pesman, E., Aydemir, C. \& Atik C. (2013) Effects of flotation deinking on the removal of main colors of oil-based inks from uncoated and coated office papers. BioResources. 8 (1), 45-58. Available from: doi: 10.15376/biores.8.1.45-58 Jingxiang, X., Jinyao, L., Haichao, L., Mingming, Z. \& Jifei, C. (2019) Research progress on water-based ink drying technology. In: IOP Conferans Series: Materials Science and Engineering. 565: 012017, 9-11 May 2019, Sanya, China. Bristol, IOP Publishing. Available from: doi: 10.1088/1757-899X/565/1/012017

Khan Akademy (2017) Electromagnetic spectrum. Available from: https://tr.khanacademy.org/science/ biology/photosynthesis-in-plants/the-light-dependent-reactions-of-photosynthesis/a/light-and-photosynthetic-pigments [Accessed 16th October 2020]

Kunwong, D., Sumanochitraporn, N. \& Kaewpirom, S. (2011) Curing behavior of a UV-curable coating based on urethane acrylate oligomer: the influence of reactive monomers. Songklanakarin Journal of Science and Technology. 33 (2), 201-207. Available from: https://rdo.psu.ac.th/ sjstweb/journal/33-2/0125-3395-33-2-201-207. pdf [Accessed 9th February 2021]

Leach, R. H., Armstrong, C., Brown, J. F., Mackenzie, M. J., Randall, L. \& Smith H. G. (1988) The Printing Ink Manual. 4th ed., Dordrecht, Netherlands, Springer, Dordrecht. Available from: doi: 10.1007/978-94-011-7097-0

McKinney, R. W. J. (1995) Technology of Paper Recycling. London, Blackie Academic \& Professional.

Mehnert, R. (1999) Excimer UV curing in printing. In: 7. International Conference on Radiation Curing, RADTECH ASIA'99, 24-26 August 1999, Kuala Lumpur, Malaysia. pp. 75-89. Available from: https:// inis.iaea.org/collection/NCLCollectionStore/_Public/31/016/31016304.pdf [Accessed 25th January 2021]

Mirković, I. B., Medek, G. \& Bolanča, Z. (2019) Ecologically sustainable printing: Aspects of printing materials. Technical Gazette. 26 (3), 662-667. Available from: doi: 10.17559/TV-20180620181128

PhotoElectronics (2019) Benefits of UV LED curing lamps. Available from: https://www. photoelcuring.com/en/technologies/uv-led/benefits-led-uvcuring-lamps/ [Accessed 20th August 2020]

PrintWiki (2018) Oxidation. Available from: http://printwiki.org/Oxidation [Accessed 5th November 2019]

Ratti, C. \& Mujumdar, A. S. (2006) Infrared drying. In: Mujumdar, In: Mujumdar, A. S. (ed.) Handbook of Industrial Drying. $3^{\text {rd }}$ Edition. Boca Raton, Florida, CRC Press, pp. 423-438.

Saad, A. A. E. R. E. (2007) Environmental pollution reduction by using VOC-free water-based gravure inks and drying them with a new drying system based on dielectric heating. PhD thesis. Bergische Universität Wuppertal.

Sappi Printer Technical Service (2012) Slow ink set \& dry. Available from: https://cdn-s3.sappi.com/ 
s3fs-public/sappietc/Slow\%20Ink\%20Set\%20 and\%20Dry.pdf [Accessed 30th November 2020]

Selim, M. S., Yesavage, V. F., Chebbi, R., Sung, S. H., Borch, J. \& Olson, J. M. (1997) Drying of waterbased inks on plain paper. Journal of Imaging Science and Technology. 41 (2), 152-158. Available from: https://www.ingentaconnect.com/ contentone/ist/jist/1997/00000041/00000002/ art00009 [Accessed 23rd December 2020]

Sharma, S. K. (2015) Paper drying by using microwaves as heating source, Apex Institute of Engineering and Technology, Jaipur, Rajasthan India. Available from: https://www.academia.edu/12390349/ Paper_Drying_By_Using_Microwaves_As_Heating_Source [Accessed 28th November 2020]

Sunnerberg, G. \& Larsson, L. O. (ed.) (1987) Paper structure and its influence upon paper-ink interaction. Topical themes in newsprint - printing research. Sweden, Swedish Newsprint Research Centre (TFL).

Smyth, S. (2003) The Print and Production Manual, Printing Inks. 9th ed. Leatherhead, United Kingdom, Pira International Ltd.

Trembley, J. F. \& Loring C. M. (1969) Drying printing inks and coatings by radio frequency heating. TAPPI, 52 (10): 1847.

Tsigonias, M., Ploumi, E., Politis, A. \& Vekinis, G. (2010) Using microwave drying systems in the graphic arts, Modern solutions for environmental industrial applications. In: Enlund, N. and Lovreček, M. (eds.) 37th International Research Conference of larigai, Advances in Printing and Media Technology. September, Montreal, Canada. Darmstadt, International Association of Research Organizations for the Information, Media and Graphic Arts Industries. pp. 291-296. Available from: http://jpmtr.org/Advances-Vol-37(2010) online.pdf [Accessed 29th December 2020].

Waghmare, R. B., Perumal, A. B., Moses, J. A. \& Anandharamakrishnan, C. (2021) Recent developments in freeze drying of foods. In: Innovative Food Processing Technologies, A Comprehensive Review. Amsterdam, Netherlands, Elsevier, pp. 82-99. Available from: doi: 10.1016/B978-0-12-815781-7.00017-2

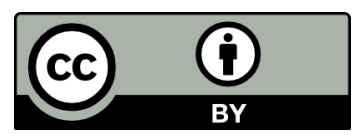

(C) 2021 Authors. Published by the University of Novi Sad, Faculty of Technical Sciences, Department of Graphic Engineering and Design. This article is an open access article distributed under the terms and conditions of the Creative Commons Attribution license 3.0 Serbia (http://creativecommons.org/licenses/by/3.0/rs/). 